OPEN

SUBJECT AREAS:

OBJECT VISION

PSYCHOLOGY

PERCEPTION

ATTENTION

Received

2 May 2013

Accepted

28 October 2013

Published

24 January 2014

Correspondence and requests for materials should be addressed to

T.Y. (yokoyama@lit. kobe-u.ac.jp)

\section{Perception of Direct Gaze Does Not Require Focus of Attention}

\author{
Takemasa Yokoyama ${ }^{1,2}$, Hiroki Sakai' ${ }^{1}$ Yasuki Noguchi' \& Shinichi Kita' \\ 'Departiment of Psychology, Kobe University, Kobe, Japan, ${ }^{2}$ Japan Society for the Promotion of Science, Japan.
}

Previous research using averted (e.g., leftward or rightward) gaze indicates that gaze perception requires a focus of attention. However, direct gaze, compared with averted gaze, is processed in the brain preferentially and enhances cognitive functions. Thus, it is necessary to use direct gaze to investigate whether gaze perception is possible without focused attention. We conducted a dual-task paradigm in which attention was drawn away from gaze. Results showed performance on gaze-direction discrimination (direct vs. averted gaze) in the dual-task condition was only slightly lower than in the single-task condition; participants were able to discriminate direct from averted gaze without focusing their attention in a similar manner to when they did focus their attention. In contrast, when participants discriminated between averted gazes (leftward and rightward), performance dropped to near-chance levels. It was concluded that gaze perception does not require a focus of attention for direct gaze.

G aze perception plays an important role in our everyday lives. The gaze of another conveys his/her desires and intentions ${ }^{1}$. In addition, individuals can predict other people's behavior by perceiving their gaze, because gaze direction signals the upcoming target or goal $^{2-7}$. Therefore, gaze perception is a significant cognitive function facilitating social interactions.

It was previously believed that gaze perception required a focus of attention. Burton et al. ${ }^{8}$ conducted an interference task to examine this notion. They set a target and a distractor of gaze or hands pointing images either leftward or rightward. The target was positioned in the center of the screen, while the distractor was positioned above or underneath the target, and they were presented simultaneously. Participants were required to attend to the target and to judge whether it indicated left or right, while ignoring the distractor. The distractor was not attended, so if it influenced the responses to the target it would have been processed without focus of attention. Results showed that gaze distractors did not influence responses to the target, but hand-pointing distractors did. The authors therefore concluded that gaze perception requires a focus of attention.

However, several previous studies of direct gaze (gaze that is directed at observers) imply that gaze perception may not require a focus of attention ${ }^{9,10}$. Direct gaze captures the viewer's spatial attention ${ }^{11-13}$ and enhances conscious awareness of faces ${ }^{14}$. Moreover, our visual systems have a cone of direct gaze, with a range of directions categorized as direct, and when the gaze of another person is hard to see, this cone expands ${ }^{15,16}$. In addition, Yokoyama, Noguchi, and Kita ${ }^{17}$ indicate that direct gaze, compared with averted gaze, is preferentially processed in the brain even when direct gaze is rendered invisible by continuous flash suppression (CFS). They also found that event-related potentials of leftward and rightward gaze are comparable when those gaze directions are rendered invisible. Burton et $\mathrm{al}^{8}{ }^{8}$ used only averted gaze (leftward and rightward gaze) and concluded that focus of attention is necessary for gaze perception, but did not examine the effects of direct gaze. It is therefore necessary to investigate whether direct gaze is perceived without a focus of attention before we conclude that a focus of attention is not required for gaze perception.

The aim of this study was to investigate whether gaze perception requires a focus of attention, using direct gaze. To this end, we used a dual-task paradigm with a gaze-direction discrimination task (direct or averted gaze). In this paradigm, participants concurrently performed two tasks: a central task and a peripheral task. The central task was an attentionally demanding task: participants needed to orient their focus of attention toward the central task while they conducted the peripheral task with little or no attentional cost. This paradigm permitted us to investigate whether a focus of attention is necessary for gaze perception, using direct gaze.

\section{Results}

Experiment 1: direct vs. averted gaze. We investigated whether a focus of attention was necessary for gaze perception and gaze-direction discrimination, using a dual-task paradigm. Participants performed the central 
attentionally demanding letter task and the peripheral gaze-direction discrimination task either concurrently or separately. We measured the role of attention to compare performance on the single task, in which attention was accessible, with performance on the dual task, in which attention was drawn away by the central task. If gaze-direction discrimination demands attention, peripheral performance in the dual-task condition should decrease significantly relative to the single-task condition. In contrast, if gaze-direction discrimination does not require many attentional resources, performances in the dual- and single-peripheral task should be comparable.

The attentionally demanding task was the letter discrimination task. Five randomly rotated letters (Ts and Ls) were presented in the center of the display and participants were required to indicate whether all letters in the display were the same or different. Previous studies have shown that this task is sufficiently effective in engaging attention away from the periphery ${ }^{18-20}$. After the letters were displayed, a facial image was presented at a random peripheral location, and participants reported whether its gaze direction was direct or averted (Fig. 1). In the dual-task condition, participants conducted both the central letter task and the peripheral gaze-direction discrimination task, while prioritizing the central letter task. All participants received training before the experiment began (see Method), and stimulus onset asynchrony (SOA) for central and peripheral tasks was determined individually for each participant during the training.

Eight participants participated in the dual-task paradigm (central letter task and/or peripheral gaze-direction discrimination task) in Experiment 1. Figure 2 shows the results from the eight participants on the single and dual task in Experiment 1. The mean ( \pm standard deviation (SD)) correct responses of the central task alone were 76.8 $\pm 3.5 \%$, while the mean correct responses of the central task in the dual-task condition were $77.3 \pm 3.4 \%$. We found no significant differences between the single- and dual-task conditions for any participant (MS: $\mathrm{t}_{18}=0.32, P=0.749$; HS: $\mathrm{t}_{18}=-0.17, P=$ 0.870; MI: $\mathrm{t}_{18}=0.40, P=0.689$; SK: $\mathrm{t}_{18}=-1.23, P=0.234$; AK: $\mathrm{t}_{18}=0.59, P=0.560$; TY: $\mathrm{t}_{18}=0.16, P=0.870$; FT: $\mathrm{t}_{18}=0.21, P=$ 0.839; TK: $\left.\mathrm{t}_{18}=1.57, P=0.134\right)$. Because those non-significant results could result from a lack of power of the t-tests, we also performed the Bayesian analysis of a null-hypothesis significant testing $^{21,22}$. When the Bayesian Information Criterion (BIC) obtained by this analysis exceeds 0.75 , the evidence is described as positive (in favor of the null hypothesis). In BIC values, six of eight participants were positive $\left(\mathrm{MS}: P_{\mathrm{BIC}}\left(\mathrm{H}_{0} \mid \mathrm{D}\right)=0.80\right.$; $\mathrm{HS}: P_{\mathrm{BIC}}\left(\mathrm{H}_{0} \mid \mathrm{D}\right)=0.81$; MI: $P_{\mathrm{BIC}}\left(\mathrm{H}_{0} \mid \mathrm{D}\right)=0.80$; SK: $P_{\mathrm{BIC}}\left(\mathrm{H}_{0} \mid \mathrm{D}\right)=0.67 ; \mathrm{AK}: P_{\mathrm{BIC}}\left(\mathrm{H}_{0} \mid \mathrm{D}\right)=0.78$; TY: $P_{\mathrm{BIC}}\left(\mathrm{H}_{0} \mid \mathrm{D}\right)=0.81$; FT: $P_{\mathrm{BIC}}\left(\mathrm{H}_{0} \mid \mathrm{D}\right)=0.81$; TK: $P_{\mathrm{BIC}}\left(\mathrm{H}_{0} \mid \mathrm{D}\right)=$ 0.57). The results of the central letter task indicated that participants' attention was focused on the central letter task during the dual-task condition. The mean correct responses of the peripheral gaze-direction discrimination task in the single- and dual-task conditions were $75.7 \pm 5.8 \%$ and $74.0 \pm 4.6 \%$, respectively. There were no significant differences between performance on the single- and dual-peripheral tasks for each participant (MS: $\mathrm{t}_{18}=0.56, P=$ 0.581; HS: $\mathrm{t}_{18}=1.73, P=0.099$; MI: $\mathrm{t}_{18}=0.45, P=0.657$; SK: $\mathrm{t}_{18}$ $=-0.19, P=0.84 ; \mathrm{AK}: \mathrm{t}_{18}=1.24, P=0.230$;Y: $\mathrm{t}_{18}=0.59, P=$ 0.564; FT: $\mathrm{t}_{18}=0.18, P=0.869$; TK: $\left.\mathrm{t}_{18}=0.40, P=0.690\right)$. In BIC

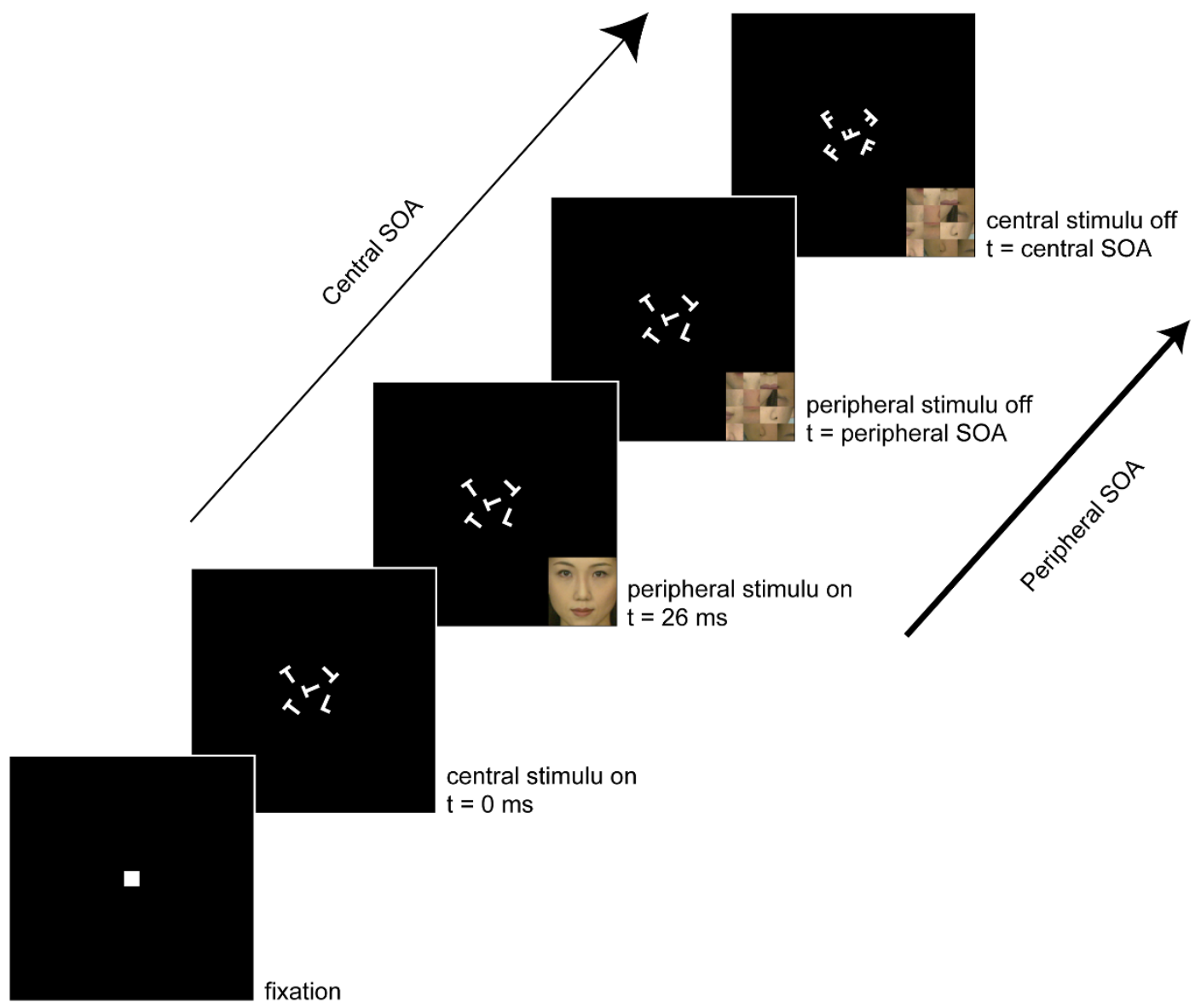

Figure $1 \mid$ An example of the sequence of events for a typical trial. Following a $300 \pm 100 \mathrm{~ms}$ fixation display, letter stimuli appeared. A peripheral facial stimulus was presented $26 \mathrm{~ms}$ after the onset of the letter stimuli. The face was then masked by a scrambled face and letter stimuli were masked by rotated letter Fs. After presentation of the trial sequence, participants indicated the gaze direction (direct or averted) of the face and/or whether the five central letters were identical (either five Ts or five Ls) or different (four Ls and one T or four Ts and one L). Central SOA and peripheral SOA denote the presentation time for letters and facial images, respectively. 
A
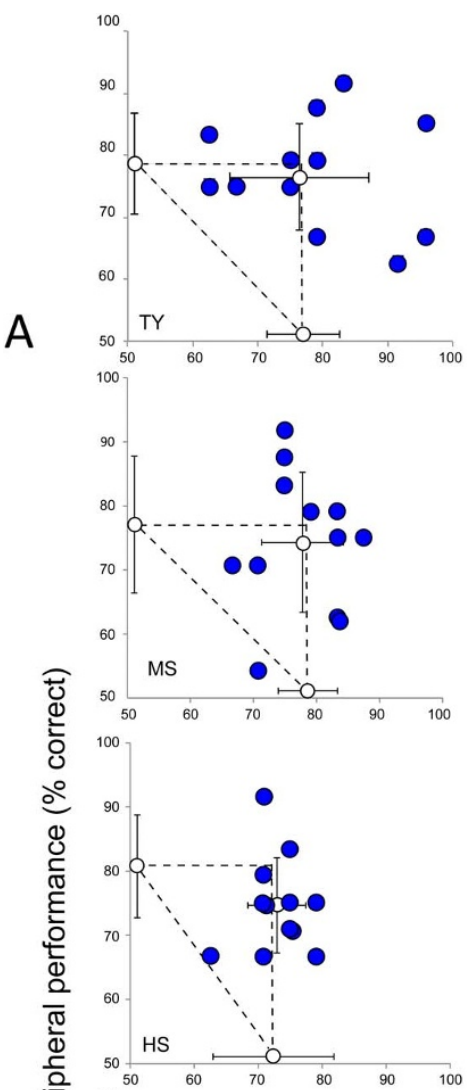

ำ

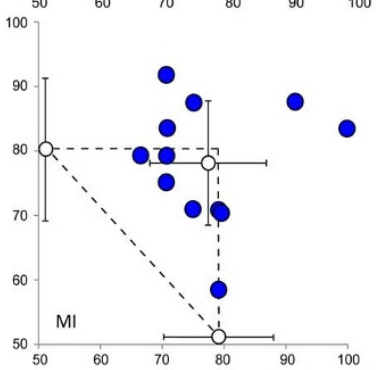

Central performance $(\%$ correct)

B

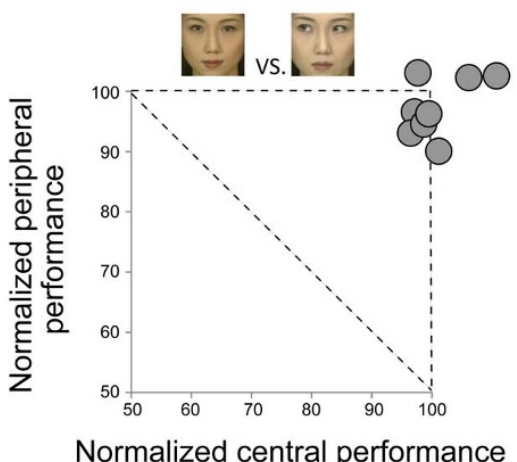

Figure $2 \mid$ Results from eight participants in Experiment 1. (A) Individual results of single- (central and peripheral) and dual-task conditions. The horizontal axis indicates the accuracy of response on the attentionally demanding central letter task, and the vertical axis indicates the accuracy of response on the peripheral gaze-direction discrimination task. Filled circles represent each participant's mean accuracy in the dual task condition (one block consisted of 24 trials). Open circles represent the mean accuracy over all blocks in the three experimental conditions (single central, single peripheral, and dual task). Error bars show the standard deviations. Mean accuracy of performance on the gaze-direction discrimination task in the dual-task condition was not significantly worse than mean accuracy of performance on the single peripheral task condition for the eight participants $(t$ test, $p>.05)$.

(B) Normalized performance values for each participant. Filled circles denote the performance for each participant in the dual-task, normalized to their single-task performance. To calculate normalized values, we used simple linear scaling which maps the mean single-task performance to $100 \%$, leaving chance at $50 \%$. Normalized performance of gaze-direction discrimination was above $90 \%$ of single-task performance for all participants. Results indicate that gaze-direction discrimination between direct and averted gaze can be performed extremely well while attentional resources are drawn away from gaze. 
values, six of eight participants were positive $\left(\mathrm{MS}: P_{\mathrm{BIC}}\left(\mathrm{H}_{0} \mid \mathrm{D}\right)=\right.$ 0.78; HS: $P_{\mathrm{BIC}}\left(\mathrm{H}_{0} \mid \mathrm{D}\right)=0.51 ; \mathrm{MI}: P_{\mathrm{BIC}}\left(\mathrm{H}_{0} \mid \mathrm{D}\right)=0.79 ; \mathrm{SK}$ : $P_{\mathrm{BIC}}\left(\mathrm{H}_{0} \mid \mathrm{D}\right)=0.81 ; \mathrm{AK}: P_{\mathrm{BIC}}\left(\mathrm{H}_{0} \mid \mathrm{D}\right)=0.67$; TY: $P_{\mathrm{BIC}}\left(\mathrm{H}_{0} \mid \mathrm{D}\right)=$ 0.78 ; FT: $P_{\mathrm{BIC}}\left(\mathrm{H}_{0} \mid \mathrm{D}\right)=0.81$; TK: $\left.P_{\mathrm{BIC}}\left(\mathrm{H}_{0} \mid \mathrm{D}\right)=0.80\right)$. In addition, the performance of all eight participants in the dual-task condition was more than $90 \%$ of their performance in the single-task condition (Fig. 2B) (see Method for calculation). Consequently, participants could effectively achieve gaze-direction discrimination even though attentional resources were not accessible for the task.

Experiment 2: inverted faces. Previous studies have indicated that inverted faces impair face and gaze perception ${ }^{13,23,24}$; this phenomenon is called the face-inversion effect. If participants perceived our stimulus set as face-like objects, rather than faces, their performance for inverted faces should be comparable with their performance on Experiment 1; otherwise, performance on the dual-task condition should decrease significantly. In addition, inverted faces can be used to control for low-level visual properties, such as contrast, luminance, and spatial frequency. If these low-level visual characteristics contributed to the results of Experiment 1, performance for inverted faces should be comparable with performance on upright faces in Experiment 1. Thus, we used inverted faces in our dual-task paradigms to investigate whether our results in Experiment 1 could be observed only when face and gaze perception occurred and could not be explained by the low-level visual features (Fig. 3A, upper).

The same eight participants were tested on the gaze-direction discrimination task with an inverted face in Experiment 2. As in Experiment 1, the results for Experiment 2 showed no significant differences between performance on the central letter task in the single- $(77.2 \pm 3.6 \%)$ and dual-task $(74.4 \pm 4.8 \%)$ conditions for all eight participants (MS: $\mathrm{t}_{18}=1.23, P=0.23$; HS: $\mathrm{t}_{18}=-0.623, P=$ 0.541; MI: $\mathrm{t}_{18}=0.64, P=0.532 ; \mathrm{SK}: \mathrm{t}_{18}=0.11, P=0.917 ; \mathrm{AK}: \mathrm{t}_{18}=$ $0.57, P=0.572$; TY: $\mathrm{t}_{18}=0.24, P=0.808$; FT: $\mathrm{t}_{18}=1.18, P=0.251$; TK: $\left.\mathrm{t}_{18}=0.98, P=0.337\right)$. In BIC values, six of eight participants were positive (MS: $P_{\mathrm{BIC}}\left(\mathrm{H}_{0} \mid \mathrm{D}\right)=0.67$; $\mathrm{HS}: P_{\mathrm{BIC}}\left(\mathrm{H}_{0} \mid \mathrm{D}\right)=0.78$; MI: $P_{\mathrm{BIC}}\left(\mathrm{H}_{0} \mid \mathrm{D}\right)=0.78$; SK: $P_{\mathrm{BIC}}\left(\mathrm{H}_{0} \mid \mathrm{D}\right)=0.81$; AK: $P_{\mathrm{BIC}}\left(\mathrm{H}_{0} \mid \mathrm{D}\right)=0.78$; TY: $P_{\mathrm{BIC}}\left(\mathrm{H}_{0} \mid \mathrm{D}\right)=0.80$; FT: $P_{\mathrm{BIC}}\left(\mathrm{H}_{0} \mid \mathrm{D}\right)=0.68$; TK: $P_{\mathrm{BIC}}\left(\mathrm{H}_{0} \mid \mathrm{D}\right)=$
0.73). The mean performance on the gaze-direction discrimination task was $73.7 \pm 6.7 \%$ when it was conducted alone, and $58.9 \pm 4.9 \%$ in the dual-task condition. Normalized performance was between $55 \%$ and $80 \%$ of the single-task levels (Fig. $3 A$, lower). There were significant differences between the single- and dual-peripheral tasks for six of the eight participants (corrected, $\mathrm{MS}$ : $\mathrm{t}_{18}=4.63, P=0.0017$; HS: $\mathrm{t}_{18}=2.07, P=0.4215 ; \mathrm{MI}: \mathrm{t}_{18}=4.11, P=0.0051$; SK: $\mathrm{t}_{18}=2.40$, $P=0.2176$; AK: $\mathrm{t}_{18}=3.62, P=0.0159$; TY: $\mathrm{t}_{18}=3.92, P=0.0080$; TK: $\left.\mathrm{t}_{18}=9.20, P=2.39 \times 10^{-7}\right)$. Furthermore, we conducted a pairwise $t$ test analysis comparing mean accuracy of performance in the inverted dual-task condition of Experiment 2 and the upright dual-task condition of Experiment 1. There were significant differences between those performances $\left(t_{7}=-6.03 ; P=0.0005\right)$. Therefore, we observed a face-inversion effect on our gaze-discrimination task. This suggests that the findings of Experiment 1 were the result of participants perceiving faces, and cannot be explained by basic low-level visual characteristics.

Experiment 3: leftward vs. rightward gaze. Next, we examined whether participants were able to discriminate leftward and rightward gaze direction without attentional resources. Burton et al. ${ }^{8}$ utilized these gaze directions to investigate whether focus of attention was necessary for gaze perception. We conducted a dualtask paradigm to examine gaze-direction discrimination between leftward and rightward gaze (Fig. 3B, upper).

The same eight participants were tested on the gaze-direction discrimination (leftward vs. rightward) task in Experiment 3. Performances on the central letter task in the single- and dual-task conditions were comparable for all eight participants (78.6 $\pm 2.9 \%$ and $76.0 \pm 3.6 \%$, respectively; $t$ test, MS: $\mathrm{t}_{18}=0.41, P=0.680 ; \mathrm{HS}_{\mathrm{t}} \mathrm{t}_{18}$ $=1.21, P=0.239 ; \mathrm{MI}: \mathrm{t}_{18}=1.16, P=0.261$; $\mathrm{SK}: \mathrm{t}_{18}=0.82, P=$ $0.424 ; \mathrm{AK}: \mathrm{t}_{18}=1.02, P=0.322 ; \mathrm{TY}: \mathrm{t}_{18}=0.24, P=0.808$; FT: $\mathrm{t}_{18}=$ $0.26, P=0.792$; TK: $\left.\mathrm{t}_{18}=0.75, P=0.462\right)$. In BIC values, five of eight participants were positive (MS: $P_{\mathrm{BIC}}\left(\mathrm{H}_{0} \mid \mathrm{D}\right)=0.80$; HS: $P_{\mathrm{BIC}}\left(\mathrm{H}_{0} \mid \mathrm{D}\right)$ $=0.68 ; \mathrm{MI}: P_{\mathrm{BIC}}\left(\mathrm{H}_{0} \mid \mathrm{D}\right)=0.69 ; \mathrm{SK}: P_{\mathrm{BIC}}\left(\mathrm{H}_{0} \mid \mathrm{D}\right)=0.75 ; \mathrm{AK}:$ $P_{\mathrm{BIC}}\left(\mathrm{H}_{0} \mid \mathrm{D}\right)=0.72$; TY: $P_{\mathrm{BIC}}\left(\mathrm{H}_{0} \mid \mathrm{D}\right)=0.80$; FT: $P_{\mathrm{BIC}}\left(\mathrm{H}_{0} \mid \mathrm{D}\right)=$ 0.80 ; TK: $\left.P_{\mathrm{BIC}}\left(\mathrm{H}_{0} \mid \mathrm{D}\right)=0.76\right)$. The average performances on the leftward and rightward gaze-direction discrimination task in the

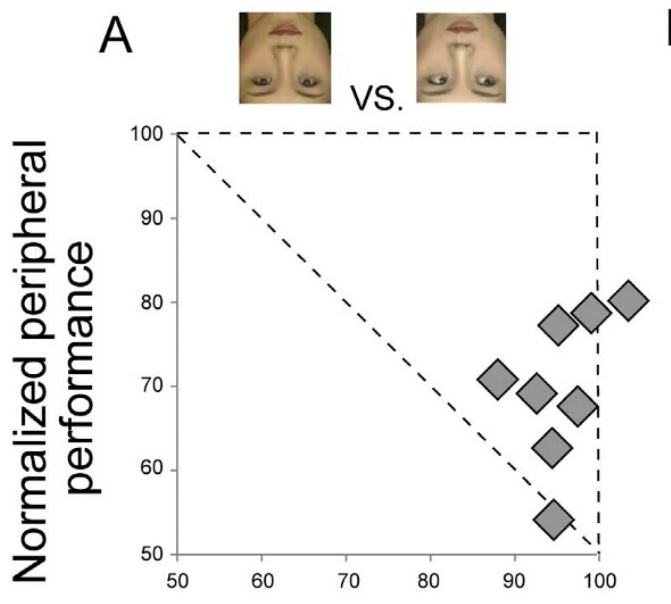

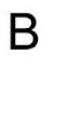

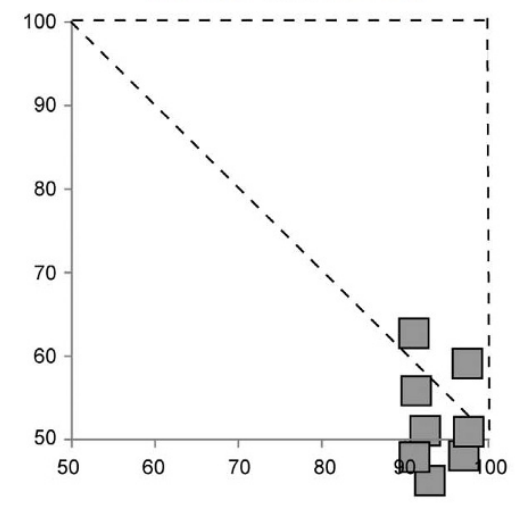

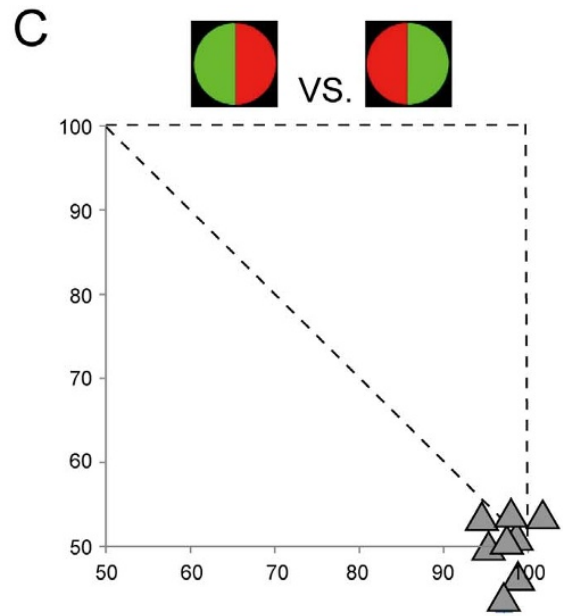

\section{Normalized central performance}

Figure 3 Normalized performance in Experiments 2-4. (A) Normalized performance on gaze-direction discrimination with inverted faces in Experiment 2. Normalized value was between $55 \%$ and $80 \%$ of the single-task levels. Performance on the dual-task condition with inverted faces was significantly lower than performance on the dual-task condition with upright faces in Experiment $1(t(7)=-6.03 ; p<.001)$. (B) Normalized performance on gaze-direction discrimination with averted gaze (leftward and rightward gaze) in Experiment 3. Normalized performance dramatically dropped to near chance level when participants discriminated leftward from rightward gaze in the dual-task condition. (C) Normalized performance on a disk-discrimination task in Experiment 4. Performance values dropped to chance levels. The results indicate that the central letter task efficiently absorbs attentional resources from the periphery. 
single- and dual-task conditions were $65.7 \pm 6.5 \%$ and $50.6 \pm 1.8 \%$, respectively. Normalized performance was $52.1 \pm 7.7 \%$ (Fig. $3 B$ ). Individually, there were significant differences between these two conditions in performance for six of the eight participants (corrected, MS: $\mathrm{t}_{18}=2.29, P=0.2749 ; \mathrm{HS}: \mathrm{t}_{18}=4.16, P=0.0047 ; \mathrm{MI}: \mathrm{t}_{18}=4.76$, $P=0.0012$; SK: $\mathrm{t}_{18}=1.01, P=1.0000 ;$ AK: $\mathrm{t}_{18}=6.11, P=7.19 \times$ $10^{-5}$; TY: $\mathrm{t}_{18}=5.01, P=0.0007 ; \mathrm{FT}: \mathrm{t}_{18}=3.10, P=0.0494 ; \mathrm{TK}: \mathrm{t}_{18}=$ $3.51, P=0.0201)$. There were no significant differences between performance on the dual-peripheral task condition and chance (50\%) for all eight participants (MS: $\mathrm{t}_{22}=-0.72, P=0.47$; HS: $\mathrm{t}_{22}$ $=0.47, P=0.639$; MI: $\mathrm{t}_{22}=-1.10, P=0.284$; SK: $\mathrm{t}_{22}=-0.70, P=$ 0.494; AK: $\mathrm{t}_{22}=-0.34, P=0.735 ; \mathrm{TY}: \mathrm{t}_{22}=0.14, P=0.888 ; \mathrm{FT}: \mathrm{t}_{22}$ $=0.35, P=0.727$; TK: $\left.\mathrm{t}_{22}=0.64, P=0.525\right)$. In BIC values, seven of eight participants were positive (MS: $P_{\mathrm{BIC}}\left(\mathrm{H}_{0} \mid \mathrm{D}\right)=0.78$; HS: $P_{\mathrm{BIC}}\left(\mathrm{H}_{0} \mid \mathrm{D}\right)=0.81 ; \mathrm{MI}: P_{\mathrm{BIC}}\left(\mathrm{H}_{0} \mid \mathrm{D}\right)=0.72$; SK: $P_{\mathrm{BIC}}\left(\mathrm{H}_{0} \mid \mathrm{D}\right)=$ 0.79; AK: $P_{\mathrm{BIC}}\left(\mathrm{H}_{0} \mid \mathrm{D}\right)=0.82 ;$ TY: $P_{\mathrm{BIC}}\left(\mathrm{H}_{0} \mid \mathrm{D}\right)=0.82$; FT: $P_{\mathrm{BIC}}\left(\mathrm{H}_{0} \mid \mathrm{D}\right)=0.82$; TK: $\left.P_{\mathrm{BIC}}\left(\mathrm{H}_{0} \mid \mathrm{D}\right)=0.79\right)$. This indicates that focus of attention is essential for discriminating leftward from rightward gaze and for the perception of averted gaze, and suggests that participants probably judged whether there was direct gaze in Experiment 1.

Experiment 4: attentionally demanding peripheral task. Finally, we tested an attentionally demanding peripheral task in Experiment 4. Our assumption was that because the central letter task engages the focus of attention in the dual-task condition, performance on the attentionally demanding peripheral task should dramatically decrease in the dual-task condition, as previous studies found ${ }^{18,20}$. We used a bisected-disk discrimination task that has previously been shown to require focus of attention ${ }^{18,20}$, to determine whether this would occur in our dual-task condition (Fig. 3C, upper).

In the central letter task, there were no significant differences in performance between single- and dual-task conditions for the eight participants $(76.4 \pm 3.2 \%$ and $75.6 \pm 2.9 \%$, respectively; $t$ test, MS: $\mathrm{t}_{38}=0.26, P=0.794 ; \mathrm{HS}: \mathrm{t}_{38}=0.07, P=0.944 ; \mathrm{MI}: \mathrm{t}_{38}=-0.54, P=$ 0.590; SK: $\mathrm{t}_{38}=0.63, P=0.532$; AK: $\mathrm{t}_{38}=0.52, P=0.600$; TY: $\mathrm{t}_{38}=$ $0.31, P=0.760$; FT: $\mathrm{t}_{38}=0.38, P=0.708$; TK: $\mathrm{t}_{38}=0.303, P=$ 0.763). In BIC values, eight participants were positive (MS: $P_{\mathrm{BIC}}\left(\mathrm{H}_{0} \mid \mathrm{D}\right)=0.86$; HS: $P_{\mathrm{BIC}}\left(\mathrm{H}_{0} \mid \mathrm{D}\right)=0.86$; MI: $P_{\mathrm{BIC}}\left(\mathrm{H}_{0} \mid \mathrm{D}\right)=$ 0.84; SK: $P_{\mathrm{BIC}}\left(\mathrm{H}_{0} \mid \mathrm{D}\right)=0.83 ; \mathrm{AK}: P_{\mathrm{BIC}}\left(\mathrm{H}_{0} \mid \mathrm{D}\right)=0.84 ; \mathrm{TY}$ : $P_{\mathrm{BIC}}\left(\mathrm{H}_{0} \mid \mathrm{D}\right)=0.85$; FT: $P_{\mathrm{BIC}}\left(\mathrm{H}_{0} \mid \mathrm{D}\right)=0.85$; TK: $P_{\mathrm{BIC}}\left(\mathrm{H}_{0} \mid \mathrm{D}\right)=$ $0.85)$. On the other hand, performance on the peripheral task in the dual-task condition $(49.8 \pm 2.5 \%)$ was significantly lower than in the single-task condition $(77.0 \pm 3.4 \%)$ for all eight participants (MS: $\mathrm{t}_{38}=13,35, P=6.31 \times 10^{-16}$; HS: $\mathrm{t}_{38}=10.87, P=3.18 \times 10^{-13}$; MI: $\mathrm{t}_{38}=8.05, P=9.78 \times 10^{-10} ; \mathrm{SK}: \mathrm{t}_{38}=8.07, P=9.18 \times 10^{-10}$; AK: $\mathrm{t}_{38}=9.84, P=5.31 \times 10^{-12}$; TY: $\mathrm{t}_{38}=11.33, P=9.51 \times 10^{-14}$; FT: $\mathrm{t}_{38}=6.86, P=6.71 \times 10^{-8}$; TK: $\left.\mathrm{t}_{38}=7.46, P=5.82 \times 10^{-9}\right)$. The normalized values shown in Figure $3 C$ indicate that performance in the dual-task condition was $49.6 \pm 5.6 \%$ of that in the single-task condition. The results of Experiment 4 indicate that the central letter task clearly absorbs attentional resources from the periphery in our dual-task conditions, and the attentionally demanding peripheral task cannot be performed under such conditions.

\section{Discussion}

In this study, we investigated whether gaze perception and gazedirection discrimination required a focus of attention for direct gaze. We conducted a dual-task paradigm in which attention was focused away from a peripheral facial stimulus. In Experiment 1, participants were able to discriminate between direct and averted gaze when attention was distracted from the facial stimulus. Thus, participants could perceive the gaze of the facial stimulus without focusing their attention on the gaze. In Experiment 2, performance on the same gaze-direction discrimination task was significantly lower when an inverted face was presented. We observed the face-inversion effect, and the results of Experiment 1 could not be attributed to low-level properties of the image set in this study. In Experiment 3, we used averted gaze to test whether participants could discriminate between leftward and rightward gazes without attentional resources. Performance on this task was close to that predicted by chance (50\%), thus indicating that a focus of attention is necessary for the perception of averted gaze. Given the results of Experiments 1 and 3, participants probably discriminated whether there was direct gaze in Experiment 1. In Experiment 4, we conducted an attentionally demanding peripheral task (discrimination between red-green and green-red disks); performance on the task dropped to chance levels when attention was not focused on the peripheral disk stimulus. Taken together, these findings indicate that gaze perception is possible without a focus of attention when the gaze direction is direct.

Our results clearly demonstrate that perception of direct gaze is possible without focus of attention, but perception of averted gaze is not. The results are consistent with several previous studies that compare direct and averted gaze. Previous studies indicate that direct gaze captures the spatial attention of viewers to a greater extent than averted gaze ${ }^{11-13}$, but see Cooper ${ }^{25}$. In addition, direct gaze enhances conscious awareness of face ${ }^{14}$. It appears that direct gaze has a higher saliency and is more preferentially processed than averted gaze before attentional modulation occurs.

The present study provided the first evidence that direct gaze could be perceived without a focus of attention. Previous studies have examined the unconscious processing of direct gaze using $\mathrm{CFS}^{14}$. Although the image of a face with direct or averted gaze was rendered invisible to one eye by continuous flashes to the other eye, the subjects' task was to detect the face presented at one of several potential positions near a fixation point. In other words, the gaze stimuli were presented at locations within a spotlight of attention, even though those stimuli were made invisible by CFS. Given the significant effect of attentional modulation reported for both invisible and visible stimuli $^{26,27}$, the attention directed to potential positions may interact with the unconscious visual processing of the invisible face, resulting in a higher sensitivity to direct gaze than to averted gaze $e^{26,27}$. The dual-task paradigm in the present study ensured that little or no attentional resource was assigned to the processing of the gaze. In addition to the large spatial separation of the central (letters) and peripheral stimuli (gaze), we imposed a highly demanding task in the central visual field. Furthermore, the location of the peripheral stimuli (gaze) was randomly varied across trials. These procedures should have prevented subjects from paying attention to the peripheral gaze in the dual-task condition. The high accuracy of the peripheral (gaze discrimination) task in both the single- and dual-task conditions of Experiment 1 thus provided stronger evidence for efficient processing of direct gaze that does not need attention.

Ricciardelli and Turatto ${ }^{28}$ have suggested that two different types of attention (input or spatial attention ${ }^{29}$ and central attention ${ }^{30}$ ) can be involved in gaze perception. While we designed our present task so that subjects could assign little or no attention to the peripheral task, it remains possible that the subjects attended both to central and peripheral stimuli simultaneously: for example, by dividing their spotlight of attention into two locations, one for central and another for peripheral stimuli ${ }^{31}$. In this sense, our experiments may not completely control spatial attention of subjects. An implication of the present study, therefore, would be that our brain could process the information of direct gaze in near absence of central attention. Additional studies are necessary for revealing whether direct gaze perception requires spatial attention.

What are the mechanisms in the brain that enable direct gaze perception without a focus of attention? We presume that the visual pathways involving subcortical structures such as the superior colliculus, pulvinar, and amygdala may underlie the higher sensitivity to direct than to averted gaze. Senju and Johnston ${ }^{8}$ proposed the firsttrack modulator model, in which the perception of direct gaze was 
mediated by the subcortical face-detection pathway. Neural processing in this pathway is considered to be so rapid that it could modulate the subsequent processing of the face stimuli via the cortical structures (e.g., the striate and extrastriate visual areas). It is thus probable that the visual information of direct gaze in the present study was also conveyed to the brain through this subcortical pathway, which enabled participants to detect it without any allocation of attention. Indeed, this mechanism of visual processing recruiting the subcortical structure has been reported for other categories of visual stimuli, such as animal images. Li et al. ${ }^{18}$ used a dual-task paradigm similar to ours, and found that subjects correctly detected animals embedded in a natural scene in the near absence of attention. Consistent with this finding, a recent study reported neurons in the human amygdala that showed a selective response to pictures of animals ${ }^{32}$.

Our findings indicate that perception of averted gaze and discrimination between leftward and rightward gaze requires a focus of attention. These results are consistent with those of Burton et al. ${ }^{8}$ and Ricciardelli and Turatto ${ }^{28}$. Burton et al. used an interference task to draw attention away from an averted gaze stimulus, and investigated whether unattended gaze influenced response to a target. They could not observe any effects of unattended gaze. In our Experiment 3 results, the normalized performance was $52.1 \pm 7.7 \%$, and there were no significant differences between performance on the dualtask condition and chance (50\%) for the eight participants. This means that participants were unable to perceive the averted gaze or to discriminate between leftward and rightward gazes. Given the results of Burton et al. and our own, it appears that unlike direct gaze, averted gaze requires a focus of attention.

The purpose of this study was to investigate whether gaze perception requires a focus of attention, using direct gaze. The ability to discriminate between direct and averted gaze when attention was focused on the task was comparable with performance when attention was diverted elsewhere. However, we could find no such effects when we used only averted gaze. It would be interesting to investigate the persistence of the effects on direct gaze. We conclude that gaze perception does not require a focus of attention when gaze is direct.

\section{Methods}

Experiment 1: direct vs. averted gaze. Participants. Eight participants, including the authors (TY and HS), were recruited from the Kobe University Department of Psychology, and were tested in all four experiments. Each participant gave informed consent after the nature of the study had been explained. All participants had normal or corrected-to-normal visual acuity. Approval for the experiment was obtained from the Ethics Committee of the Department of Psychology, Kobe University, Kobe, Japan.

Apparatus and stimuli. Visual stimuli were displayed on a Sony MultiScan 17 sf II 14.1-inch CRT display with a resolution of $1,024 \times 768$ pixels. Displays and data collection were controlled with MATLAB, using the Psychophysics toolbox ${ }^{33,34}$, on a Dell Optiplex 360 computer with Microsoft Windows XP (the refresh rate was $75 \mathrm{~Hz}$ ). We used six pictures (three male and three female; $3.0^{\circ} \times 3.0^{\circ}$ of visual angle) from the ATR DB99 database (ATR Promotions, Kyoto, Japan), and all faces had neutral expressions. The gaze direction of each person pictured was leftward, straight, and rightward. We created a mask stimulus for the faces, which was a scrambled face consisting of all facial parts except the eyes.

Procedure. Figure 1 shows an example of a trial sequence. Each trial used a fixation display (300 \pm 100$)$, followed by letter stimuli (Ts and Ls) in the center of the display. A peripheral facial stimulus was presented $26 \mathrm{~ms}$ after the onset of the central letter stimuli. The peripheral facial stimulus was masked by a scrambled face, then the central letter stimuli were masked by rotated letter Fs, at an angle that corresponded to the previously displayed Ts and/or Ls. After the masked display, the response display appeared, and participants were required to respond to the central letter task and/or the peripheral gaze-direction discrimination task as accurately as possible. During the task, participants were instructed to fixate on the center of the screen. Beep sounds were provided as feedback when participants responded incorrectly. An experimental block consisted of 24 trials. Participants performed eight blocks in the single-task condition (central and peripheral tasks), and 12 blocks in the dual-task condition. Participants were tested individually in a dark room. A chin-rest was used to maintain a distance of approximately $57 \mathrm{~cm}$ from the CRT display.
Central letter discrimination task. The central letter task was conducted as the attentionally demanding task. In this task, we used five randomly rotated Ts and Ls, either all identical or one different from the other four (four Ts and one L, or four Ls and one T). Those letters appeared at the center of the screen at nine possible locations within $1.2^{\circ}$ of the visual angle. Participants judged whether all five letters were the same. The SOA was set individually for each participant and varied between 186 and $240 \mathrm{~ms}$.

Peripheral gaze-direction discrimination task (direct or averted). The peripheral task was a gaze-direction discrimination task. A facial image was presented at a random location centered on the edge of an imaginary rectangle subtending $8.0^{\circ} \times 10.0^{\circ}$ of the visual angle. Participants indicated whether the gaze direction of the facial image was direct (straight gaze) or averted (leftward or rightward gaze). The SOA of the peripheral gaze-direction discrimination task was set individually for each participant and varied between 160 and $186 \mathrm{~ms}$.

Dual task. Participants were required to perform both the central and peripheral tasks in the dual-task condition. The SOAs of both tasks were the same as those for the single-task conditions for each participant.

We calculated normalized performances for the dual-task condition, using a simple linear scaling of the mean accuracy of each participant's performance. The scaling plotted mean accuracy of the single-task condition to $100 \%$, leaving chance at $50 \%$ :

$$
\text { Normalized performance }=0.5+0.5\left[\left(P_{2}-0.5\right) /\left(P_{1}-0.5\right)\right],
$$

where $P_{1}$ and $P_{2}$ refer to performance in the single- and dual-task conditions, respectively.

Training. Before each experiment began, all participants received training. The central task used in training was the same as that in the main experiment (letter task); however, the peripheral task used in training was a sex-judgment task, rather than the gaze-direction discrimination task. We used 100 pictures of faces (50 male and 50 female; Softpia Japan Foundation, Gifu, Japan) for training. At the beginning of the training, the SOAs of the central and peripheral tasks were $506 \mathrm{~ms}$ and $186 \mathrm{~ms}$, respectively. When mean accuracy exceeded $90 \%$ during the 24 -trial block, the SOAs were decreased independently for both tasks. The central task SOA was decreased to less than $250 \mathrm{~ms}$ for all participants to avoid the possibility of eye movements. Training ended when the SOA of the central task had stabilized below $250 \mathrm{~ms}$. Training lasted between 8 and 12 hours for each participant.

Experiment 2: inverted faces. We inverted the facial stimuli used in Experiment 1 by rotating each face $180^{\circ}$ along the horizontal axis (Fig. $3 A$, upper).

Experiment 3: leftward vs. rightward gaze. Gaze directions in the peripheral task were leftward and rightward in Experiment 3. Participants judged whether the gaze direction of a face was leftward or rightward.

Experiment 4: attentionally demanding peripheral task. We used a vertically bisected disk with red and green halves and another similar disk rotated by $180^{\circ}$. The disk size was $1.5^{\circ} \times 1.5^{\circ}$ of the visual angle. A mask stimulus for the disk was a disk divided into four red and green alternating quadrants. In the peripheral task, participants reported whether the disk was red-green or green-red (Fig. 3C, upper). Participants performed 12 blocks in the single-task condition (central and peripheral tasks) and 24 blocks in the dual-task condition. To determine the SOA of the diskdiscrimination task, participants received one hour of training. The SOA in the peripheral task varied between 80 and $93 \mathrm{~ms}$.

1. Baron-Cohen, S. Mindblindness: an essay on autism and theory of mind. (MIT Press, 1995).

2. Galfano, G. et al. Eye gaze cannot be ignored (but neither can arrows). Q J Exp Psychol 65, 1895-1910 (2012).

3. Itier, R. J., Villate, C. \& Ryan, J. D. Eyes always attract attention but gaze orienting is task-dependent: evidence from eye movement monitoring. Neuropsychol $\mathbf{4 5}$, (2007).

4. Langton, S. R., Watt, R. J. \& Bruce, I. I. Do the eyes have it? Cues to the direction of social attention. Trends Cog. Sci 4, 50-59 (2000).

5. Law, A. S., Langton, S. R. H. \& Logie, R. H. Assessing the impact of verbal and visuospatial working memory load on eye-gaze cueing. Vis Cogn 18, (2010).

6. Vecera, S. P. \& Rizzo, M. Eye gaze does not produce reflexive shifts of attention: evidence from frontal-lobe damage. Neuropsychol 44, 150-159 (2006).

7. Yokoyama, T., Noguchi, Y. \& Kita, S. Attentional shifts by gaze direction in voluntary orienting: evidence from a microsaccade study. Exp. Brain Res. 223, 291-300 (2012).

8. Burton, A. M., Bindemann, M., Langton, S. R., Schweinberger, S. R. \& Jenkins, R. Gaze perception requires focused attention: evidence from an interference task. J. Exp. Psychol. Hum. Percept. Perform. 35, 108-118 (2009).

9. Conty, L., N'Diaye, K., Tijus, C. \& George, N. When eye creates the contact! ERP evidence for early dissociation between direct and averted gaze motion processing. Neuropsychol. 45, 3024-3037 (2007).

10. Senju, A. \& Johnson, M. H. The eye contact effect: mechanisms and development. Trends Cog. Sci. 13, 127-134 (2009). 
11. Conty, L., Tijus, C., Hugueville, L., Coelho, E. \& George, N. Searching for asymmetries in the detection of gaze contact versus averted gaze under different head views: a behavioural study. Spat. Vis. 19, 529-545 (2006).

12. von Grunau, M. \& Anston, C. The detection of gaze direction: a stare-in-thecrowd effect. Percept 24, 1297-1313 (1995)

13. Yokoyama, T., Ishibashi, K., Hongoh, Y. \& Kita, S. Attentional capture by change in direct gaze. Percept. 40, 785-797 (2011).

14. Stein, T., Senju, A., Peelen, M. V. \& Sterzer, P. Eye contact facilitates awareness of faces during interocular suppression. Cognit. 119, 307-311 (2011).

15. Mareschal, I., Calder, A. J. \& Clifford, C. W. G. Humans Have an Expectation That Gaze Is Directed Toward Them. Curr. Biol. 23, 717-721 (2013).

16. Mareschal, I., Calder, A. J., Dadds, M. R. \& Clifford, C. W. G. Gaze categorization under uncertainty: Psychophysics and modeling. J. Vis. 13, 1-10 (2013).

17. Yokoyama, T., Noguchi, Y. \& Kita, S. Unconscious processing of direct gaze: Evidence from an ERP study. Neuropsychol. 51, 1161-1168 (2013).

18. Li, F. F., VanRullen, R., Koch, C. \& Perona, P. Rapid natural scene categorization in the near absence of attention. Proc. Natl. Acad. Sci. USA 99, 9596-9601 (2002).

19. Reddy, L. \& Koch, C. Face identification in the near-absence of focal attention. Vis. Res. 46, 2336-2343 (2006).

20. Reddy, L., Wilken, P. \& Koch, C. Face-gender discrimination is possible in the near-absence of attention. J. Vis. 4, 106-117 (2004)

21. Masson, M. E. A tutorial on a practical Bayesian alternative to null-hypothesis significance testing. Behav. Res. Methods 43, 679-690 (2011).

22. Wetzels, R. \& Wagenmakers, E. J. A default Bayesian hypothesis test for correlations and partial correlations. Psychon. B. Rev. 19, 1057-1064 (2012).

23. Farah, M. J., Tanaka, J. W. \& Drain, H. M. What causes the face inversion effect? J. Exp. Psychol. Hum. Percept. Perform. 21, 628-634 (1995).

24. Freire, A., Lee, K. \& Symons, L. A. The face-inversion effect as a deficit in the encoding of configural information: direct evidence. Percept 29, 159-170 (2000).

25. Cooper, R. M., Law, A. S. \& Langton, S. R. H. Looking back at the stare-in-the crowd effect: Staring eyes do not capture attention in visual search. J. Vis. 13, 11-22 (2013).

26. Bahrami, B., Carmel, D., Walsh, V., Rees, G. \& Lavie, N. Spatial attention can modulate unconscious orientation processing. Percept 37, 1520-1528 (2008).

27. Shin, K., Stolte, M. \& Chong, S. C. The effect of spatial attention on invisible stimuli. Atten. Percept. Psychophys. 71, 1507-1513 (2009).

28. Ricciardelli, P. \& Turatto, M. Is attention necessary for perceiving gaze direction? It depends on how you look at it: Evidence from the locus-of-slack method. Vis. Cogn. 19, 154-170 (2011).

29. Posner, M. I. Orienting of attention. Q. J. Exp. Psychol. 32, 3-25 (1980).
30. Johnston, J. C., McCann, R. S. \& Remington, R. W. Chronometric evidence for two types of attention. Psychol. Sci. 6, 365-369 (1995).

31. Muller, M. M., Malinowski, P., Gruber, T. \& Hillyard, S. A. Sustained division of the attentional spotlight. Nature 424, 309-312 (2003).

32. Mormann, F. et al. A category-specific response to animals in the right human amygdala. Nat. Neurosci. 14, 1247-1249 (2011).

33. Brainard, D. H. The Psychophysics Toolbox. Spat. Vis. 10, 433-436 (1997)

34. Pelli, D. G. The VideoToolbox software for visual psychophysics: transforming numbers into movies. Spat. Vis. 10, 437-442 (1997).

\section{Acknowledgments}

We greatly thank participants in this study. We also thank our laboratory members for support in carrying out this work. This study was supported by a grant to the first author (T.Y.) from the Grant-in-Aid for JSPS Fellows [11J02271], the third author (Y.N.) from the Grant-in-Aid for Young Scientists (A) [22680022], and the fourth author (S.K.) from the Strategic Information and Communications R\&D Promotion Programme (SCOPE) [101707012].

\section{Author contributions}

Conceived the experiments: T.Y. Designed the experiments: T.Y., H.S., Y.N., S.K. Performed the experiments: T.Y., H.S. Analyzed the data: T.Y., H.S. Contributed reagents/ materials/analysis tools: T.Y., H.S., Y.N., S.K. Wrote the main manuscript text: T.Y. Prepared figures: T.Y. All authors reviewed the manuscript.

\section{Additional information}

Competing financial interests: The authors declare no competing financial interests.

How to cite this article: Yokoyama, T., Sakai, H., Noguchi, Y. \& Kita, S. Perception of Direct Gaze Does Not Require Focus of Attention. Sci. Rep. 4, 3858; DOI:10.1038/srep03858 (2014)

This work is licensed under a Creative Commons Attribution-

NonCommercial-NoDerivs 3.0 Unported license. The images in this article may not be included in the article's Creative Commons license. If you wish to re-use any of these image obtain permission from the license holder as indicated in the article. To view a copy of this license, visit http://creativecommons.org/licenses/by-nc-nd/3.0 


\section{SCIENTIFIC \\ REP RTS}

DOI: 10.1038/srep04318

SUBJECT AREAS: ERRATUM: Perception of Direct Gaze Does Not Require Focus of Attention

OBJECT VISION

PSYCHOLOGY

Takemasa Yokoyama, Hiroki Sakai, Yasuki Noguchi \& Shinichi Kita

PERCEPTION

ATTENTION

This Article contains an error in Figure 2a. The "TY" plot was repeated in the bottom right plot and should be data corresponding to "TK". The correct Figure 2a appears below as Figure 1.

SCIENTIFIC REPORTS:

$4: 3858$

DOI: $10.1038 /$ srep03858

(2014)

Published:

24 January 2014

Updated: 12 March 2014
A

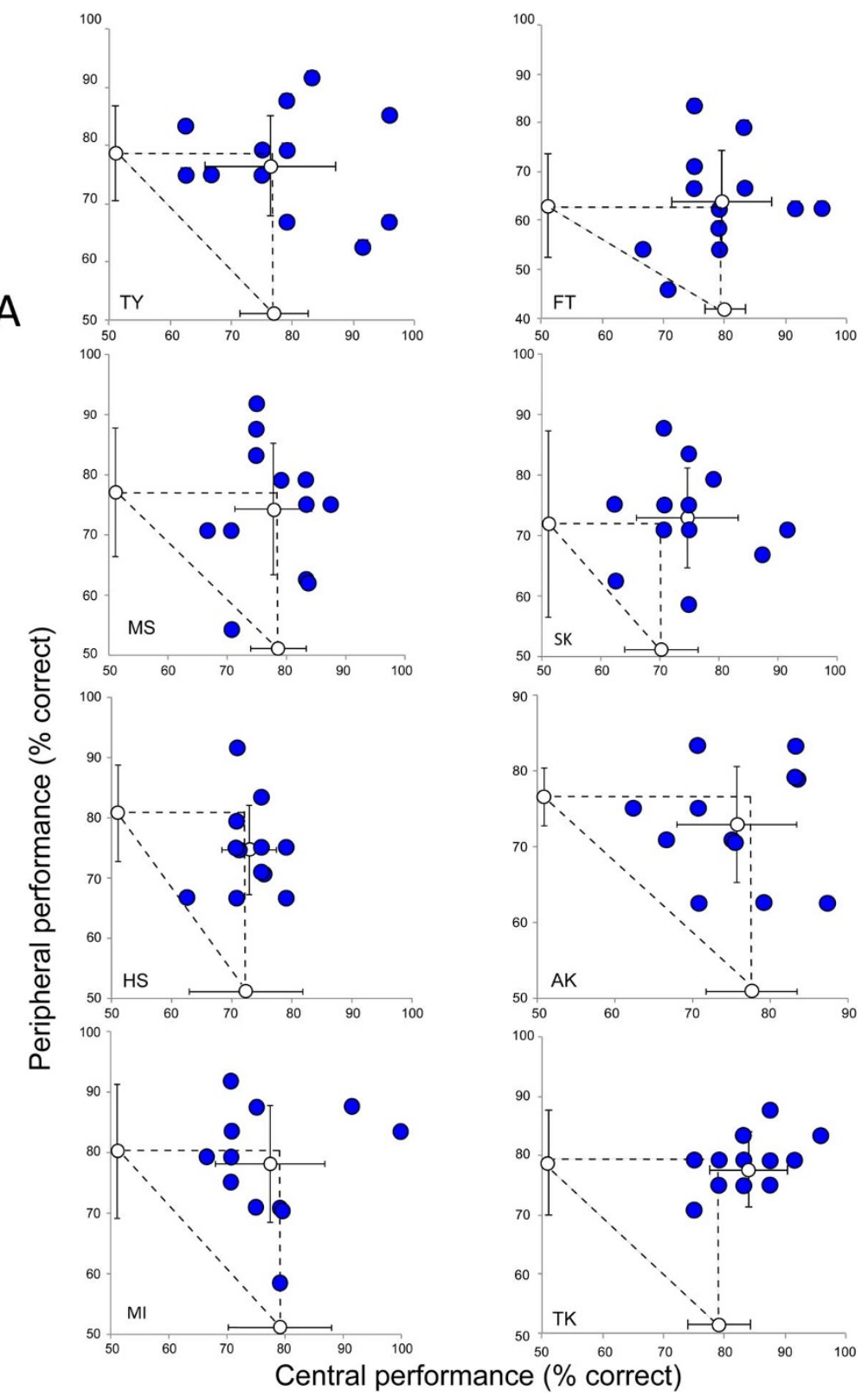

Figure $1 \mid$ 